\title{
A state-of-the-art calculation of atmospheric lepton fluxes
}

\author{
Anatoli Fedynitch* \\ DESY, Platanenallee 6, 15738 Zeuthen, Germany \\ E-mail: anatoli.fedynitch@desy.de
}

Hans Dembinski

Max Planck Institute for Nuclear Physics, Saupfercheckweg 1, 69117 Heidelberg, Germany

\section{Ralph Engel}

Karlsruher Institut für Technologie, Institut für Kernphysik, Postfach 3640, 76021 Karlsruhe, Germany

\section{Thomas K. Gaisser}

Bartol Research Institute, Department of Physics and Astronomy, University of Delaware,

Newark, DE 19716, USA

\section{Felix Riehn}

LIP Lisbon, Av. Prof. Gama Pinto 2, 1649-003 Lisbon, Portugal

\section{Todor Stanev}

Bartol Research Institute, Department of Physics and Astronomy, University of Delaware, Newark, DE 19716, USA

\begin{abstract}
Atmospheric leptons are of continuous interest for several scientific communities. At low and intermediate energies, atmospheric neutrinos are the signal for studies of fundamental neutrino properties and at high energies, they constitute the background for the characterization of the features of the astrophysical neutrino flux. We have studied in depth the relation between observable inclusive lepton fluxes/ratios and hadronic interactions by using a novel numerical transport equation solver MCEq. It enables us to characterize, with an unprecedented precision, relevant particle interaction energies, important hadron species, and the secondary particle production phase-space in terms of typical accelerator observables. Based on our findings, we improved existing interaction models SIBYLL and DPMJET and created an efficient scheme to propagate correlated model uncertainties into derived lepton fluxes. We present state-of-the-art calculations of inclusive lepton fluxes and discuss possible directions towards reducing uncertainties below $10 \%$.
\end{abstract}

35th International Cosmic Ray Conference - ICRC2017

10-20 July, 2017

Bexco, Busan, Korea

${ }^{*}$ Speaker. 


\section{Introduction}

Atmospheric muons and neutrinos are copiously created in extensive air showers that emerge from interactions of cosmic rays with the atmosphere. Spanning many orders of magnitude, from $\mathrm{MeV}$ to hundreds of $\mathrm{PeV}$, the flux of these leptons can be employed as a beam for studies of fundamental neutrino properties at low energies. At high energies, atmospheric neutrinos constitute the background for the identification of astrophysical neutrinos. At lower energies, conventional atmospheric leptons come mostly from decays of charged pions and kaons. Above several tens of $\mathrm{TeV}$, the so called prompt leptons originate from decays of short-lived, heavy-flavor mesons, such as $\mathrm{D}^{+}, \mathrm{D}^{0}, \mathrm{D}_{\mathrm{s}}^{+}+$c.c.

This contribution concentrates on our latest results regarding the uncertainties of our calculations. The most sophisticated investigation of theoretical uncertainties of atmospheric fluxes is the study by Barr et al. [1], where the authors discussed the main sources of errors: 1) from modeling of hadronic interactions and 2) from the parameterization of the cosmic ray flux. The study was tailored for the relevant energy ranges of that time, the energy range of Super-K, and was ultimately limited by the Monte Carlo method, which becomes inefficient at high energies. The hadronic uncertainty estimates were derived by propagating weights for each neutrino type, based on the production properties of the mother meson. Separate regions in particle production phase space were defined, based on the secondary energy fraction and the energy of the nucleon-air collision, which correspond roughly to the availability of experimental data and the underlying physical process. Depending on the observable, errors were claimed not to exceed 30\% below $1 \mathrm{TeV}$ and are of the order of $10 \%$ at lower energies. While at low energies pion production is relatively well studied at fixed-target experiments and thus results in smaller error estimates, at high energies the absence of full phase-space kaon measurements on light nuclear targets dominates the prediction error. This has not changed until today, and therefore, the results of [1] are still valid.

What has changed, though, is the knowledge of the primary cosmic ray fluxes, which is the second largest source of uncertainty. Motivated by the availability of new direct and indirect cosmic ray measurements and the typically conservative approaches to cosmic ray flux uncertainties, we developed a new data-driven approach to parametrize the elemental flux, the composition and the error on the fluxes cosmic ray nuclei in the range between $10 \mathrm{GV}$ and $10^{12} \mathrm{GeV}$. The new model is called Global Spline Fit (GSF) and is discussed in more detail in a different contribution [2]. Some features of GSF relevant for the current contribution are also given in Section 2.

So, why is a new calculation of uncertainties needed? First, the pioneering work [1] had to neglect correlations between the different phase space regions and symmetries between those regions wrt. particle and anti-particle production, which we can technically handle in the new scheme. Secondly, the hadronic interaction model in [1], TARGET, relies completely on data and includes a minimal fraction of the underlying theory, making the extrapolation to high energies less certain, than for example by using SiBYLL 2.3 [3]. We employ full hadronic interaction models, which are used as event generators for air shower simulations and physics at colliders. Thirdly, by using GSF we can reduce the cosmic ray flux errors to a minimum and disentangle the ambiguity between the hadronic and cosmic ray uncertainties. The combination of these advantages will allow us to invert the exercise and use inclusive atmospheric lepton measurements, some of which exceed the prediction in terms of precision, to constrain the extrapolation of hadronic interaction models [4], 
or, for the first time to constrain hadronic model parameters directly and less ambiguously. Further, the technological advancement of neutrino detectors promises significant reduction of systematic uncertainties of measurements in intermediate energy ranges, e.g. [5], which will be ultimately limited by theory at some point. Finally, the origin of astrophysical neutrinos is still a widely open question. A better estimate of the expected conventional and prompt neutrino backgrounds (better would be its detection), would allow for more solid statements about the properties of the diffuse flux, thus enabling theorists to narrow the number of candidates in multi-messenger approaches.

\section{Calculation method and models}

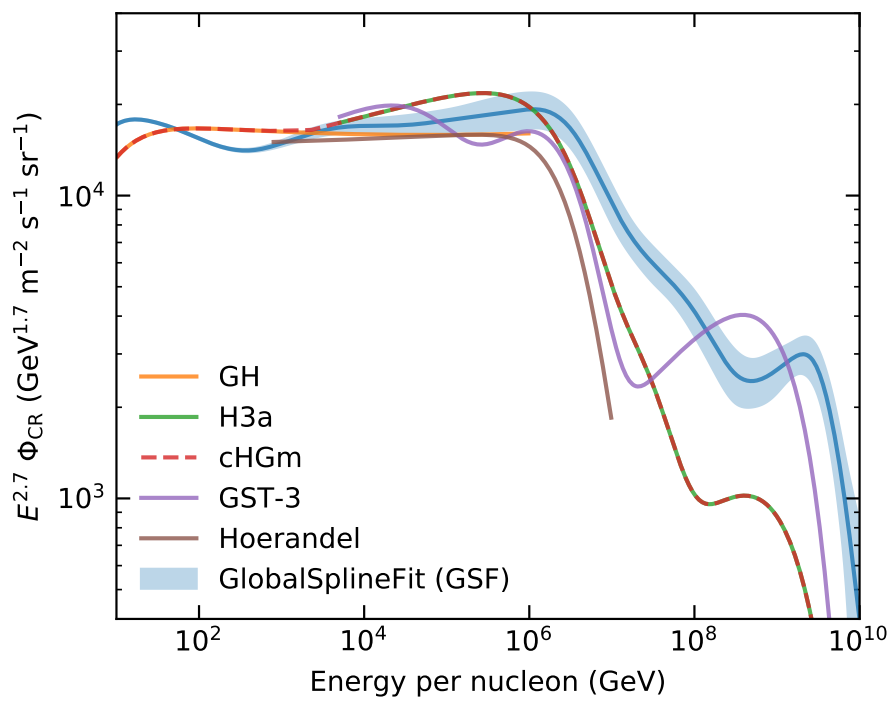

Figure 1: Nucleon fluxes at the top of the atmosphere. The new spline fit (GSF) is accompanied by a $1 \sigma$ error band. Each flux model is shown in the energy range for which it was designed for. The numerical implementation of the shown models $[6,7,8,9,10]$ (in descending order) are available in CRFLUXMoDELS, an MCEQ sub-package.

For the calculation of the fluxes we use our open-sourced numerical solver MCEQ ${ }^{1}$. The code has been outlined in [11] and more details will be published later this year. To summarize, the method is based on the direct numerical solution of the transport equations for particles in the Earth's atmosphere, the hadronic coupled cascade equations. A comprehensive overview of how to approximate the solution analytically is described in [12] and publications therein. The numerical solution has some advantages: it permits non power-law primary spectra, a curved geometry and arbitrary shapes of air density profiles. In addition, there is direct access to tables of inclusive differential cross sections for secondary particle production, which can be modified directly, in run-time, without the need for recomputing $Z$ factor integrals.

We use a new model of the cosmic ray flux, which is based on fitting splines to the available cosmic ray measurements (details will be discussed presented in [2]). Popular cosmic ray flux models are superimposed in Figure 1. GSF is a significant departure from the previous parameterizations above the knee, while below the knee, the error band tends to cover the model variations. In

\footnotetext{
${ }^{1}$ https://github.com/afedynitch/MCEq
} 
the sub-TeV region, the fit is mainly driven by recent direct observations. As a consequence of the hardening of the proton and helium spectra, it disagrees with the shape of the 'GH' model, which has been used in all low energy neutrino flux calculations as the base-line model. The GSF errors on the nucleon flux are a few percent below $10 \mathrm{TeV}$ and increase to $\gtrsim 15 \%$ up to the highest energies, where it blows up due a poorly known proton fraction. As we will see, the shape difference between knee and ankle strongly influences the prompt flux predictions.

Hadronic interactions are modeled using inclusive particle distributions obtained by directly running Monte Carlo event generators. Since some of the programs become inaccurate below $\sqrt{s} \sim 10 \mathrm{GeV}$, we chose DPMJET-III [13] to extend the coverage to lower energies. While DPMJET contains the necessary physical models, its performance has not been recently verified at these energies, thus leading to potential inaccuracy below $\sim 30 \mathrm{GeV}$ lepton energy. The transition occurs at $80 \mathrm{GeV}$ via smooth interpolation of secondary particle yields. On longer term, the calculation will be improved by using better low energy models. Below $5-10 \mathrm{GeV}$ lepton energy, further effects, such as the geomagnetic cutoff and 3D enhancement become relevant, which are also not included here. Although both shortcomings affect the absolute value of the predicted flux, the uncertainty estimates are not expected to significantly change.

\section{Uncertainties of inclusive atmospheric lepton fluxes and ratios}

Figure 2 displays our results on top of measurements. All post-LHC models are compatible with each other within the error bands. The error bands obtained for each of the models separately agree to a high degree, except for SIB YLL-2.1. The hadronic uncertainties shown here are universal and by changing the hadronic interaction model, only the "central value" of the prediction changes but not its relative error. Vertical muons (top left panel) are measured with a better precision than the prediction error. The cosmic ray flux uncertainty in this energy range is very small driven, again, by the AMS-02 results. Thus, the remaining differences can be attributed to the hadronic model and the atmosphere. For this calculation the atmosphere is fixed to the location of L3+c [14] using the NRLMSISE-00 model [19]. A part of the mismatch with the BeSS-TeV data, can be attributed to this choice. The new SIB YLL-2.3C [20] is somewhat low, which we can confirm by comparing it to accelerator distributions. The comparison to the horizontal MUTRON result in the top right panel, probes pion production at higher energies, with smaller influence from additional kaon decays, since pion absorption is less pronounced for more inclined showers. The errors are statistical only and the absence of systematic uncertainty estimates reduces the significance of this comparison.

Our muon neutrino predictions are well within experimental errors (lower left panel). At low energies, neutrino oscillations influence the Super-K measurement, which are not included in the calculations. At higher energies, our favored model SIBYLL-2.3C lies directly on top of the ANTARES measurement, which is a remarkable result, given that we didn't tune the model to any atmospheric lepton observables. In the range $10 \mathrm{GeV}$ to $10 \mathrm{TeV}$, Epos-LHC [21], QGSJETII-04 [22] and SiBYLL-2.3C approximately coincide with the HKKMS'15 [23] reference model. The different shapes around $100 \mathrm{GeV}$ can be mostly attributed to GSF.

The electron neutrino flux is experimentally not well known at high energies and our calculation unsurprisingly agrees with the available data. At the very high energy, one can clearly see the 

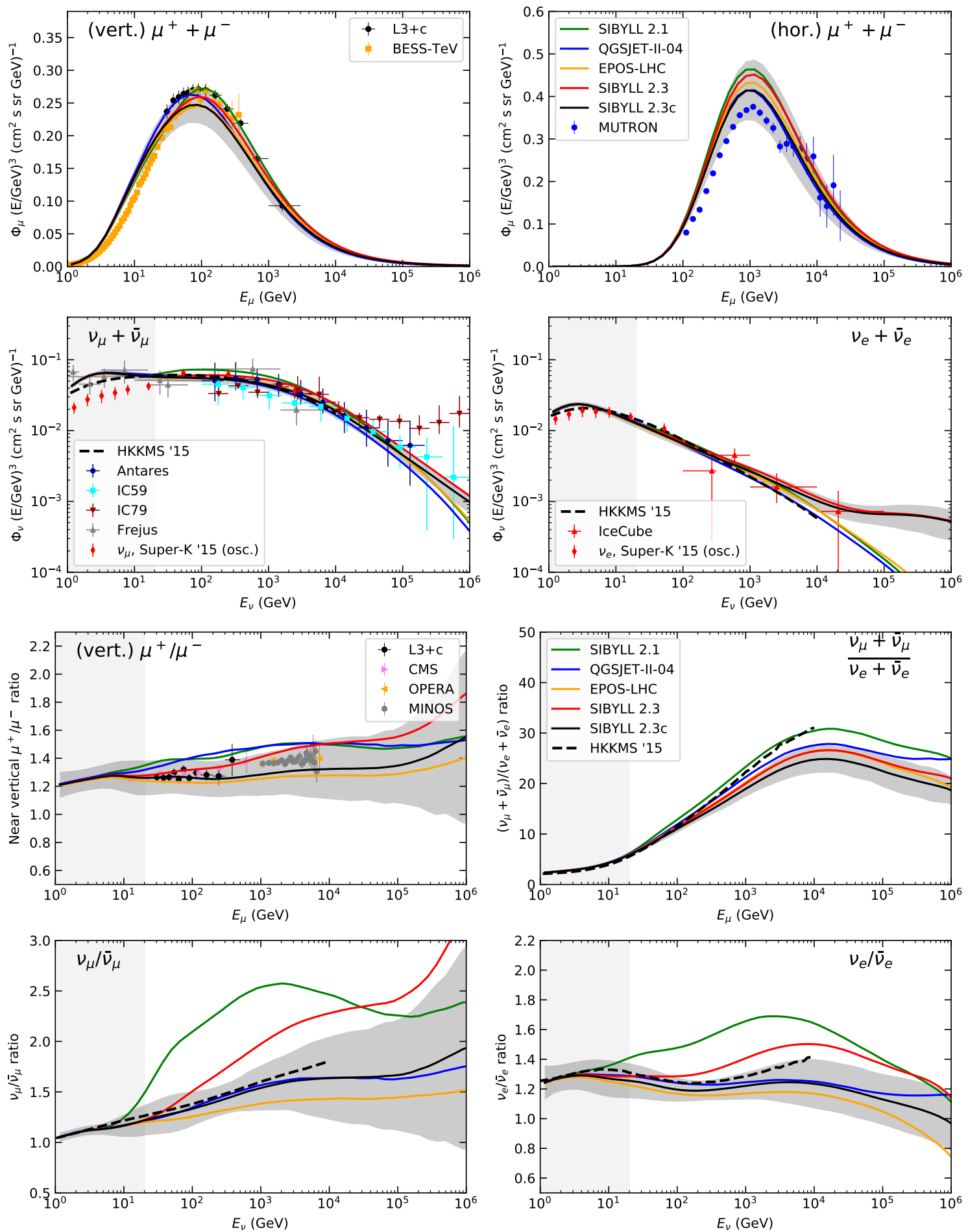

Figure 2: Atmospheric lepton flux (upper panels) and particle ratios (lower panels). The gray error band belongs to the SIBYLL-2.3C line. Each hadronic model is surrounded by a very similar band, which is omitted for clarity. Experimental data from [4, 14, 15, 16, 17, 18]. 
transition from the conventional to the prompt flux for both SIBYLL-2.3 models. At lower energies, the shape differs from the HKKMS' 15 prediction, mostly due to the hardening of the proton and helium spectra in the GSF cosmic ray flux model.

The situation on ratios is less clear from the experimental perspective. The only particleantiparticle ratio that is well measured is the muon charged ratio. As it can be clearly seen in the top left panel, the precision of the measurements exceeds the theoretical uncertainty. This fact will allow us to constrain the very uncertain associated production of $\mathrm{K}^{+}$in $\mathrm{p}+\mathrm{A} \rightarrow \Lambda+\mathrm{K}^{+}+\mathrm{X}$, which is one of the largest uncertainties for conventional lepton fluxes at high energies, where muons more abundantly come from kaon decays. OPERA have shown [4] how their measurement of the muon charge ratio in the $\mathrm{TeV}$ range constrains associated production. The mismatch in the description of the muon charge ratio at high energies is extremely challenging to fix when developing hadronic interaction models based on fixed-target observations only, since interaction energies are close to $\sim 100 \mathrm{TeV}$ and it is unclear how to extrapolate the associated production.

The neutrino ratios in the other panels reveal one of the reasons why we found it necessary to improve SibYLL-2.3, by releasing the "c" version. From a detailed inspection of available kaon fixed-target data, we concluded that the $\mathrm{K}^{+}$production as implemented in version 2.3 tends to be overestimated, however not for the same reason as for SIBYLL-2.1. We released therefore version $2.3 \mathrm{c}$, that restores forward Feynman scaling behavior with minimal impact on all other air shower observables or charm production, and has improved treatment of kaons. We find that the HKKMS'15 calculation up to its highest energies is covered by our uncertainty band.
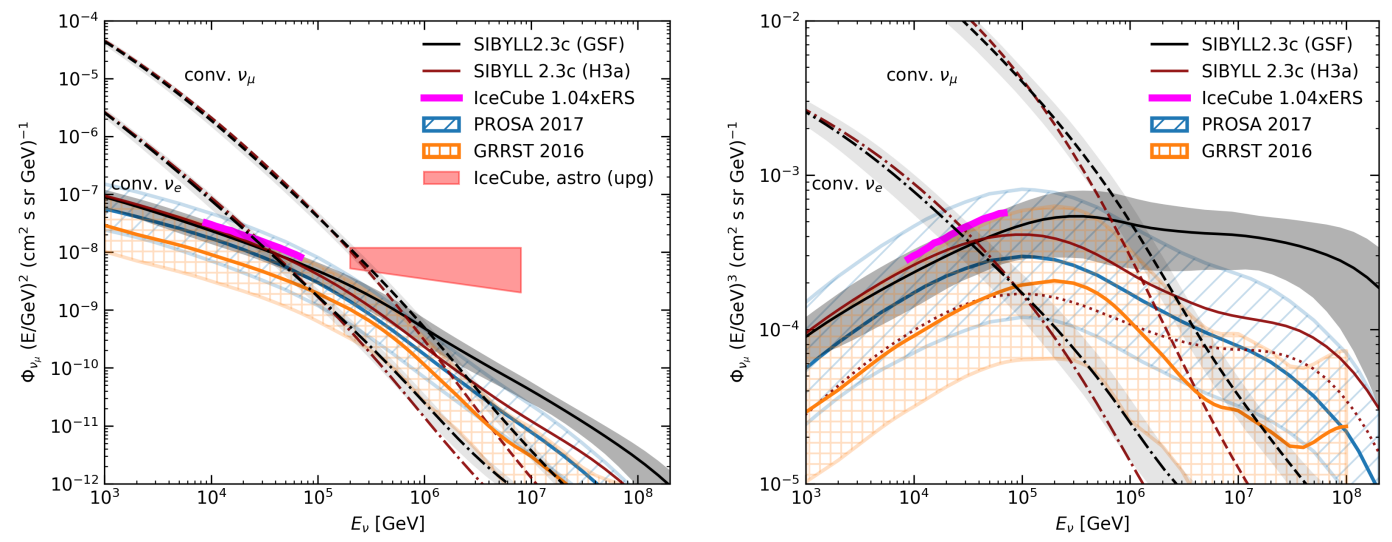

Figure 3: Prompt lepton fluxes scaled with $E^{2}$ (left) and $E^{3}$ (right). Our uncertainty bands include combined hadronic and cosmic ray errors. PROSA [24] and GRRST [25] calculations are shown in their H3a versions and include all QCD uncertainties. The SIBYLL-2.3c prediction is separately shown for H3a and the new GSF, while the dotted line represents the perturbatively produced charm only. IceCube band and prompt limit from [26].

One of the key features of SIBYLL-2.3 is the production of charmed hadrons that allows us to estimate prompt lepton production in air showers and thus in inclusive lepton calculations. We have outlined some of the model ideas in the past $[3,27]$. Although the model for perturbative charm production is less sophisticated than in recent calculations of prompt leptons, as for example those in Figure 3, having charm production in SIBYLL has some advantages. First, the model is 
capable of full air shower simulations and therefore, it allows us to include the prompt component in detector simulations, and second, it contains models for non-perturbative charm production, which are needed to describe low-energy, fixed-target data.

The remarkable feature of our new calculation is that due to the GSF flux, the spectral index above few tens of $\mathrm{TeV}$ deviates from calculations with $\mathrm{H} 3 \mathrm{a}$ or similar models. However, within the prompt error bands, all models are still compatible. From the left panel one can guess, that the shape differences might have an impact on astrophysical neutrino analyses, derived from statistical arguments [26]. By inspecting the differences between the dotted and the SIBYLL-2.3C H3a line in the right panel, one can estimate the importance of the non-perturbative components.

\section{Conclusion}

We have presented a subset of the latest results of our inclusive flux calculations, focusing on the new primary flux model GSF, the updated SIBYLL-2.3c and uncertainties. GSF provides a consistent error estimate for the cosmic-ray fluxes over the entire energy range. It has a large impact at high energy, modifying the expectation of the prompt neutrino fluxes. The improved kaon production in the new SIB YLL-2.3c release solves a subtle model feature that impacted atmospheric muon and neutrino calculations. Finally, we demonstrated the calculation of hadronic uncertainties following the approach of [1] using a fast, fully numerical scheme, which is slightly improved by extending it to higher energies and by including a similar uncertainty estimation for charmed particles. One key observation is that recent interaction models are compatible within the errors, and that lepton error bands obtained from this scheme do not depend on the choice of the hadronic interaction model.

\subsection{Acknowledgments}

AF thanks for funding from the European Research Council (ERC) under the European Union's Horizon 2020 research and innovation programme (Grant No. 646623). TG and TS acknowledge support from the U.S. National Science Foundation (PHY-1505990).

\section{References}

[1] G. D. Barr, T. K. Gaisser, S. Robbins, and T. Stanev, Uncertainties in atmospheric neutrino fluxes, Phys. Rev. D74 (2006) 094009, [astro-ph/ 0611266 ].

[2] H. Dembinski, R. Engel, A. Fedynitch, T. K. Gaisser, F. Riehn, and T. Stanev, Data-driven model of the cosmic-ray flux and composition, Proceedings of the ICRC 2017, Busan, Korea, 12 - 20 July 2017.

[3] F. Riehn, R. Engel, A. Fedynitch, T. K. Gaisser, and T. Stanev, A new version of the event generator Sibyll, PoS ICRC2015 (2016) 558, [arXiv: 1510 . 0 0 56].

[4] OPERA Collaboration, N. Agafonova et al., Measurement of the TeV atmospheric muon charge ratio with the complete OPERA data set, Eur. Phys. J. C74 (2014) 2933, [arXiv:1403.0244].

[5] IceCube Collaboration, M. G. Aartsen et al., Search for sterile neutrino mixing using three years of IceCube DeepCore data, Phys. Rev. D95 (2017), no. 11 112002, [arXiv: 1702 . 0516].

[6] T. K. Gaisser and M. Honda, Flux of atmospheric neutrinos, Ann. Rev. Nucl. Part. Sci. 52 (2002) 153-199, [hep-ph/0203272].

[7] T. K. Gaisser, Spectrum of cosmic-ray nucleons, kaon production, and the atmospheric muon charge ratio, Astropart. Phys. 35 (2012) 801-806, [arXiv: 1111. 6675]. 
[8] A. Fedynitch, J. B. Tjus, and P. Desiati, Influence of hadronic interaction models and the cosmic ray spectrum on the high energy atmospheric muon and neutrino flux, arXiv:1206.6710.

[9] T. K. Gaisser, T. Stanev, and S. Tilav, Cosmic Ray Energy Spectrum from Measurements of Air Showers, Front. Phys.(Beijing) 8 (2013) 748-758, [arXiv: 1303.3565$].$

[10] J. R. Hörandel, On the knee in the energy spectrum of cosmic rays, Astropart. Phys. 19 (2003) 193-220, [astro-ph/0210453].

[11] A. Fedynitch, R. Engel, T. K. Gaisser, F. Riehn, and T. Stanev, Calculation of conventional and prompt lepton fluxes at very high energy, EPJ Web Conf. 99 (2015) 08001, [arXiv: 1503.0054 ].

[12] T. K. Gaisser, R. Engel, and E. Resconi, Cosmic Rays and Particle Physics. Cambridge University Press, 2016.

[13] S. Roesler, R. Engel, and J. Ranft, The monte carlo event generator DPMJET-III, Proc. Advanced Monte Carlo for Radiation Physics, Lisbon, Portugal (2000).

[14] L3 Collaboration, P. Achard et al., Measurement of the atmospheric muon spectrum from 20-gev to 3000-gev, Phys. Lett. B598 (2004) 15-32, [hep-ex/ 0408114$].$

[15] ANTARES Collaboration, S. Adrian-Martinez et al., Measurement of the atmospheric $v_{\mu}$ energy spectrum from $100 \mathrm{GeV}$ to $200 \mathrm{TeV}$ with the ANTARES telescope, Eur. Phys. J. C73 (2013), no. 10 2606, [arXiv:1308 .1599]. [Eur. Phys. J.C73,2606(2013)].

[16] IceCube Collaboration, M. G. Aartsen et al., Measurement of the Atmospheric $v_{e}$ Spectrum with IceCube, Phys. Rev. D91 (2015) 122004, [arXiv: 1504.0375$].$

[17] IceCube Collaboration, M. G. Aartsen et al., Measurement of the $v_{\mu}$ energy spectrum with IceCube-79, arXiv:1705.0778.

[18] Frejus Collaboration, K. Daum et al., Determination of the atmospheric neutrino spectra with the Frejus detector, Z. Phys. C66 (1995) 417-428.

[19] J. M. Picone, A. E. Hedin, D. P. Drob, and A. C. Aikin, NRLMSISE-00 empirical model of the atmosphere: Statistical comparisons and scientific issues, Journal of Geophysical Research (Space Physics) 107 (Dec., 2002) 1468.

[20] F. Riehn, R. Engel, A. Fedynitch, T. K. Gaisser, and T. Stanev, The hadronic interaction model SIB YLL 2.3C and Feynman scaling, Proceedings of the ICRC 2017, Busan, Korea, 12 - 20 July 2017.

[21] T. Pierog, I. Karpenko, J. M. Katzy, E. Yatsenko, and K. Werner, EPOS LHC: Test of collective hadronization with data measured at the CERN Large Hadron Collider, Phys. Rev. C92 (2015), no. 3 034906, [arXiv:1306.0121].

[22] S. Ostapchenko, Monte Carlo treatment of hadronic interactions in enhanced Pomeron scheme: I. QGSJET-II model, Phys. Rev. D83 (2011) 014018, [arXiv: 1010 . 1869].

[23] M. Honda, M. Sajjad Athar, T. Kajita, K. Kasahara, and S. Midorikawa, Atmospheric neutrino flux calculation using the NRLMSISE-00 atmospheric model, Phys. Rev. D92 (2015), no. 2 023004, [arXiv:1502.0391].

[24] PROSA Collaboration, M. V. Garzelli et al., Prompt neutrino fluxes in the atmosphere with PROSA parton distribution functions, JHEP 05 (2017) 004, [arXiv: 1611.0381 ].

[25] R. Gauld, J. Rojo, L. Rottoli, S. Sarkar, and J. Talbert, The prompt atmospheric neutrino flux in the light of LHCb, JHEP 02 (2016) 130, [arXiv: 1511 . 0634 ].

[26] IceCube Collaboration, M. G. Aartsen et al., Observation and Characterization of a Cosmic Muon Neutrino Flux from the Northern Hemisphere using six years of IceCube data, Astrophys. J. 833 (2016), no. 1 3, [arXiv:1607.0800].

[27] F. Riehn, R. Engel, A. Fedynitch, T. K. Gaisser, and T. Stanev, Charm production in SIBYLL, EPJ Web Conf. 99 (2015) 12001, [arXiv:1502.0635]. 